\title{
Radonification Problem for Cylindrical Measures on Tensor Products of Banach Spaces
}

By

Neven ELEZović**

\begin{abstract}
Abstrace
An operator $w_{1} \otimes w_{2}$ is said to be $p$-Radonifying if it maps every cylindrical measure of type $p$, defined on the tensor product $E \otimes F$ of two Banach spaces, into a Radon probability cf order $p$ on the completion of some normed product $G \otimes_{a} H$. In this paper we prove that $w_{1} \otimes w_{2}$ is $p$-Radonifying, $1<p<\infty$, if and only if it is $\widetilde{p}$-summing.
\end{abstract}

\section{§. Introduction}

The Radonification problem for cylindrical measures on Banach spaces has been studied by A. Badrikian, S. Chevet, B. Maurey, Y. Okazaki, L. Schwartz and others, cf. e.g. [1], [11], [12] and in the references stated there. In the Schwartz's approach to this problem one try to find all operators $w$ : $E \rightarrow G$ which map every cylindrical measure on $E$ of type $p$ into a Radon probability on $G$ of order $p$. Such operators are called $p$-Radonifying. The main result is: for $1<p<\infty, w$ is $p$-Radonifying if and only if it is $p$-summing. For $0 \leqslant p \leqslant 1$, the situation is more complex.

B. Maurey considered in [5] a class of $F$-cylindrical probabilities on $E \otimes F$, which lies somewhere between cylindrical measures on $E \otimes F$ and probabilities on some completion of this space (nearer to the first ones). $\mathrm{He}$ tries to find $(p, F)$-Radonifying operators $W: E \otimes F \rightarrow G \otimes F$ of the form $W=w \otimes 1_{F}$, which map every $F$-cylindrical probability on $E \otimes F$ of type $(p, F)$ into a Radon probability on some completion $G \hat{\otimes}_{\alpha} F$ of the space $G \otimes F$. It turns out that $(p, F)$ summing operators are $(p, F)$-Radonifying for $1<p<\infty$, under some additional assumptions on the norm $\alpha$ and on the space $F$ (cf. [5], Exposé II, Théoréme 2). As an example, it is shown that if $w: E \rightarrow G$ is $p$-summing, then $w \otimes 1_{F}$ :

Communicated by S. Matsuura, September 24, 1985.

* Elektrotehnički fakuitet, Unska 3, P.B. 170, 41001 Zagreb, Yugoslavia. 
$E \otimes F \rightarrow G \hat{\otimes}_{\varepsilon} F$ is $(p, F)$-summing. Also, if $w$ is $p$-left-nuclear, then $w \otimes 1_{F}$ : $E \otimes F \rightarrow G \hat{\otimes}_{d_{p}} F$ is ( $\left.p, F\right)$-summing (cf. [2], Proposition 5 and [3]. Theorem 3).

In this paper we give an analogous result for the class of cylindrical measures on $E \otimes F$ of type $p$. It will be shown that $\tilde{p}$-summing operators of the form $w_{1} \otimes w_{2}$ are $p$-Radonifying, for $1<p<\infty$.

Let $1<p<\infty$. By classical methods it is easy to obtain that an operator of the form $1_{E} \otimes w: E \otimes F \rightarrow E \otimes H$, where $w: F \rightarrow H$ is $p$-summing, maps every cylindrical measure on $E \otimes F$ of type $p$ into an $H$-cylindrical probability on $E \otimes H$ of type $(p, H)$. Thus, Maurey's result shows that the tensor product $w_{1} \otimes w_{2}$ of two $p$-summing operators is $p$-Radonifying, from $E \otimes F$ into $G \hat{\otimes}_{\mathrm{g}} H$, under additional assumption of reflexivity of the space $H$.

The direct approach which we use in this paper gives something more. Namely, [2] Theorem 3 shows that the product $w_{1} \otimes w_{2}$ of two $p$-summing operators is $\tilde{p}$-summing from $E \otimes F$ into $G \hat{\otimes}_{\alpha} H$, whenever $\alpha$ satisfies $\alpha \leqslant / d_{p}$ or $\alpha \leqslant g_{p} \backslash$. Thus, Theorem 6.3 states that such operator is $p$-Radonifying from $E \otimes F$ in $G \hat{\otimes}_{\alpha} H, \alpha \leqslant / d_{p}$ or $\alpha \leqslant g_{p} \backslash$, without assumption on the reflexivity of the space $H$.

$\S 2$ is preparatory. In $\S 3$ we define cylindrical measures on $E \otimes F$ and establish the connection between them and probabilities on some completion $E \hat{\otimes}_{\alpha} F$. Other necessary notions (type, convergence, Fourier transform, image of cylindrical measure by an operator of the form $w_{1} \otimes w_{2}$, etc.) are introduced in $\S 4$ and $\S 5$. The main theorem, announced before, is proved in $\S 6$.

\section{§2. Notation and some Preliminary Results}

Throughout this paper $E, F, G, H$ will denote real Banach spaces, $E^{\prime}$, $F^{\prime}, G^{\prime}, H^{\prime}$ their topological duals. $\mathcal{L}(E, G)$ stands for the space of all continuous linear operators: $E \rightarrow G$. By $\left[u, x^{\prime}\right] \in F$ we denote the action of an element $u \in E \otimes F$ on vectors in $E^{\prime}$. The element $u \in E \otimes F$ induces a finitedimensional linear operator $\hat{u}: E^{\prime} \rightarrow F$ by $\hat{u} x^{\prime}:=\left[u, x^{\prime}\right] .\langle\circ, \circ\rangle$ will always denote the canonical pairing, in various settings, e.g., for $x^{\prime} \in E^{\prime}, y^{\prime} \in F^{\prime}$ and $u \in E \otimes F$ it holds $\left\langle u, x^{\prime} \otimes y^{\prime}\right\rangle=\left\langle\left[u, x^{\prime}\right], y^{\prime}\right\rangle=\left\langle\hat{u} x^{\prime}, y^{\prime}\right\rangle$.

Let $\left\{x_{j}\right\}$ be a sequence in $E$. By $N_{p}\left(x_{j}\right)$ we denote the number, finite or not

$$
N_{p}\left(x_{j}\right):= \begin{cases}\left\{\sum_{j}\left\|x_{j}\right\|^{p}\right\}^{1 / p} & , 1 \leqslant p<\infty \\ \sup _{j}\left\|x_{j}\right\| & , p=\infty\end{cases}
$$

and by 


$$
M_{p}\left(x_{j}\right):=\sup \left\{N_{p}\left(\left\langle x_{j}, x^{\prime}\right\rangle\right),\left\|x^{\prime}\right\| \leqslant 1\right\}
$$

For $\left\{u_{j}\right\} \subset E \otimes F$ (and similarly for $\left\{v_{j}\right\} \subset \mathcal{L}(E, G)$ ) we denote

$$
\begin{aligned}
& Q_{p}\left(u_{j}\right):=\sup \left\{N_{p}\left(\left\langle u_{j}, x^{\prime} \otimes y^{\prime}\right\rangle\right),\left\|x^{\prime}\right\| \leqslant 1,\left\|y^{\prime}\right\| \leqslant 1\right\} \\
& S_{p}\left(u_{j}\right):=\sup \left\{N_{p}\left(\left[u_{j}, x^{\prime}\right]\right),\left\|x^{\prime}\right\| \leqslant 1\right\}
\end{aligned}
$$

Linear operators $w: E \rightarrow G, W: E \otimes F \rightarrow G$ for which it exists a constant $C \geqslant 0$ such that

$$
\begin{aligned}
& N_{p}\left(w x_{j}\right) \leqslant C \quad M_{p}\left(x_{j}\right) \\
& N_{p}\left(W\left(u_{j}\right)\right) \leqslant C \quad S_{p}\left(u_{j}\right) \\
& N_{p}\left(W\left(u_{j}\right)\right) \leqslant C \quad Q_{p}\left(u_{j}\right)
\end{aligned}
$$

for all finite sets $\left\{x_{1}, \cdots, x_{n}\right\}$ in $E$ or $\left\{u_{1}, \cdots, u_{n}\right\}$ in $E \otimes F$, are called $p$-summing, $(p, F)$-summing and $\tilde{p}$-summing (respectively). The infimum of all constants $C$ in (2.1)-(2.3) is denoted by $\pi_{p}(w), \pi_{p, F}(W), \tilde{\pi}_{p}(W)$, respectively.

It is known that $\tilde{p}$-summing operators: $E \otimes F \rightarrow G$ are $(p, F)$-summing, and also a $p$-summing from $E \otimes_{\alpha} F$ into $G$, for arbitrary reasonable norm $\alpha$ (cf. [2], Proposition 1) and hence continuous from $E \otimes_{\varepsilon} F$ into $G$, with the norm

$$
\|W\| \leqslant \widetilde{\pi}_{p}(W)
$$

( $\varepsilon$ denotes the least reasonable crossnorm).

\section{§3. Cylindrical Measures on $\mathbb{E} \otimes$ and $\mathbb{R a d o n}$ Probabilities on $\mathbb{E} \hat{\bigotimes}_{\omega} \mathbb{F}$}

By $F C(E)$ we denote the family of all closed subspaces in $E$ of the finite codimension. The canonical projection $E \rightarrow E / N, N \in F C(E)$ is denoted by $\pi_{N}$, the projections $E / N_{1} \rightarrow E / N_{2}, N_{1} \subset N_{2}$ by $\pi_{N_{2} N_{1}}$. A cylindrical measure on $E$ is a projective system $\left\{\lambda_{N}, \pi_{N}, N \in F C(E)\right\}$ of Radon probabilities on finite-dimensional quotients of the space $E$; for $N_{1} \subset N_{2}$ it holds $\lambda_{N_{2}}=\pi_{N_{2} N_{1}}\left(\lambda_{N_{1}}\right)$. It is well known that such system defines a finitely additive measure $\lambda$ on the algebra of cylindrical sets in $E$, by

$$
\lambda(B):=\lambda_{N}\left(B_{N}\right)
$$

where $B=\pi_{N}^{-1}\left(B_{N}\right)$, and $B_{N}$ is a Borel set in $E / N$. We denote $\lambda=\left(\lambda_{N}\right)$.

For convenience, we denote $\pi_{N \otimes M}:=\pi_{N} \otimes \pi_{M}: E \otimes F \rightarrow(E / N) \otimes(F / M)$, $N \in F C(E), M \in F C(F)$, and by $\pi_{N_{2} N_{1} \otimes M_{2} M_{1}}:=\pi_{N_{2} N_{1}} \otimes \pi_{M_{2} M_{1}}:\left(E / N_{1}\right) \otimes\left(F / M_{1}\right) \rightarrow$ $\left(E / N_{2}\right) \otimes\left(F / M_{2}\right), N_{1} \subset N_{2}, M_{1} \subset M_{2}$.

The following is obvious: 
Proposition 3.1. If $N_{1} \subset N_{2}, M_{1} \subset M_{2}$ are closed subspaces of the finite codimension, then the following diagram commutes:

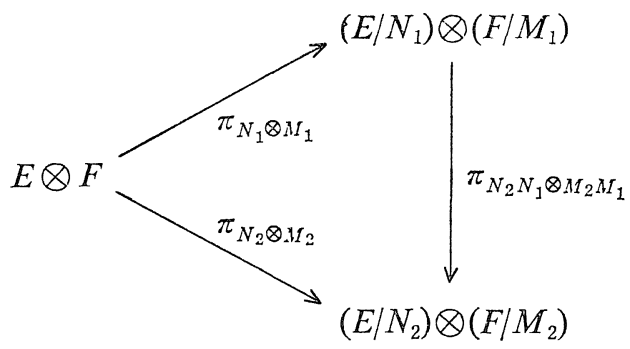

Definition. A cylindrical measure $\lambda$ on $E \otimes F$ is a projective system $\left\{\lambda_{N \otimes M}\right.$, $\left.\pi_{N \otimes M}, N \in F C(E), M \in F C(F)\right\}$ of the Radon probabilities on finite dimensional spaces $(E / N) \otimes(F / M)$.

$\mathscr{M}^{c}(E \otimes F)$ stands for the space of all cylindrical measures on $E \otimes F$.

The cylindrical algebra on a vector space $X$ depends only on the dual pair $\left(X, X^{\prime}\right)$, and remains the same if the original topology on $X$ is replaced by another which gives the same dual. More generally, if $(X, Y)$ is a pair of vector spaces in duality, such that $Y$ separates points in $X$, then the cylindrical algebra on $X$ depends only on the space $Y$.

$\left(E \otimes F, E^{\prime} \otimes F^{\prime}\right)$ is a pair of vector spaces in separated duality. Hence, we can define the cylindrical algebra on $E \otimes F$ not introducing any topology on $E \otimes F . \quad$ A cylinder is a set of the form

$$
C=\left\{u \in E \otimes F:\left(\left\langle u, u_{j}^{\prime}\right\rangle\right)_{1 \leqslant j \leqslant n} \in B\right\}
$$

where $n \in \mathbb{N}, u_{1}^{\prime}, \cdots, u_{n}^{\prime} \in E^{\prime} \otimes F^{\prime}$ and $B$ is a set in the Borel algebra $\mathscr{B}\left(\boldsymbol{R}^{n}\right)$.

It is not quite obvious that cylindrical measure can measure the cylinders! Namely, sets $C$ of the form (3.2) need not be of the form $\left\{u \in E \otimes F: \pi_{N \otimes M}(u)\right.$ $\in B\}$ for some $N \in F C(E), M \in F C(F)$, so we must prove that $\lambda(C)$ is (well) defined. For the sequel, it will be sufficient to prove

Proposition 3.2. Let $\lambda$ be a cylindrical measure on $E \otimes F$. For $u^{\prime} \in E^{\prime}$ $\otimes F^{\prime}$ and $B \in \mathscr{B}(\mathbb{R})$, the measure

$$
u^{\prime}(\lambda)(B):=\lambda\left\{u \in E \otimes F:\left\langle u, u^{\prime}\right\rangle \in B\right\}
$$

is well defined.

Proof. Take a representation $u^{\prime}=\sum_{j=1}^{n} \xi_{j}^{\prime} \otimes \eta_{j}^{\prime}$ where $n$ is minimal with this property. Then, $\left\{\xi_{1}^{\prime}, \cdots, \xi_{n}^{\prime}\right\}$ and also $\left\{\eta_{1}^{\prime}, \cdots, \eta_{n}^{\prime}\right\}$ are linearly independent 
(cf. [10], Lemma 1.2). Denote $N^{0}:=\operatorname{span}\left\{\xi_{1}^{\prime}, \cdots, \xi_{n}^{\prime}\right\} \subset E^{\prime}, M^{0}:=\operatorname{span}\left\{\eta_{1}^{\prime}\right.$, $\left.\cdots, \eta_{n}^{\prime}\right\} \subset F^{\prime}$, and let $N:=\left(N^{0}\right)^{0} \subset E, M:=\left(M^{0}\right)^{0} \subset F$ be their polars. By Auerbach lemma, there exists a basis $\left\{x_{1}^{\prime}, \cdots, x_{n}^{\prime}\right\}$ for $N^{0}$ and a basis $\left\{\bar{x}_{1}, \cdots, \bar{x}_{n}\right\}$ for $E / N \simeq\left(N^{0}\right)^{\prime}$ such that $\left\langle x_{i}^{\prime}, \bar{x}_{j}\right\rangle=\delta_{i j}$ (cf. [6], p. 22). Similarly, there exists $\left\{y_{1}^{\prime}, \cdots, y_{n}^{\prime}\right\} \subset M^{0}$ and $\left\{\bar{y}_{1}, \cdots, \bar{y}_{n}\right\} \subset F / M$ with the same properties. Then it holds $\pi_{N}=\sum_{j=1}^{n} x_{j}^{\prime} \otimes \bar{x}_{j}, \pi_{M}=\sum_{k=1}^{n} y_{k}^{\prime} \otimes \bar{y}_{k}$, and $u^{\prime}$ has a representation $u^{\prime}=\sum_{j, k=1}^{n} t_{j k}$ $x_{j}^{\prime} \otimes y_{k}^{\prime}$.

Define now $\rho:(E / N) \otimes(F / M) \rightarrow \mathbb{R}$ by $\rho\left(\bar{x}_{j} \otimes \bar{y}_{k}\right):=t_{j k} . \quad \rho$ is continuous linear mapping, and it holds

$$
\begin{aligned}
\left(\rho \circ \pi_{N \otimes M}\right)(u) & =\rho\left(\sum_{j, k}\left\langle u, x_{j}^{\prime} \otimes y_{k}^{\prime}\right\rangle \bar{x}_{j} \otimes \bar{y}_{k}\right) \\
& =\sum_{j, k} t_{j k}\left\langle u, x_{j}^{\prime} \otimes y_{k}^{\prime}\right\rangle=\left\langle u, u^{\prime}\right\rangle
\end{aligned}
$$

for every $u \in E \otimes F$. Thus, $u^{\prime}(\lambda)=\left(\rho \circ \pi_{N \otimes M}\right)(\lambda)=\rho\left(\lambda_{N \otimes M}\right)$ is well defined probability on $\mathbb{R}$.

In the classical situation, every probability (normed $\sigma$-additive measure on the Borel $\sigma$-algebra $\mathcal{B}(E)$ ) on a separable Banach space defines a cylindrical measure (finitely additive measure on the smaller cylindrical algebra) in the unique way. Moreover, for a given cylindrical measure $\lambda$ on the cylindrical algebra $\mathcal{A}(E)$, there exists at most one probability $\mu$ such that $\mu \mid \mathcal{A}(E)=\lambda$. The necessary and sufficient condition for the existence of $\mu$ is the $\sigma$-additivity of $\lambda$. The reason for this uniqueness lies in the fact that $\mathcal{B}(E)$ is the $\sigma$-algebra generated by $\mathcal{A}(E)$. Even in the non-separable case, a cylindrical measure defines at most one Radon probability on $E$ ([11], p. 174), the tightness property of Radon probabilities becomes now essential.

The connection between cylindrical measures on $E \otimes F$ and Radon probabilities on some completion $E \hat{\otimes}_{\alpha} F$ of this space is more complex. If we wish to obtain an one-to-one correspondence between them, we need some additional conditions on the norm $\alpha$. Namely, the cylindrical algebra on $E \otimes F$ is far away from the cylindrical algebra on $E \hat{\otimes}_{\alpha} F$, the first one is considerably smaller.

Let us suppose that $\alpha$ satisfies

$$
\left|\left\langle u, x^{\prime} \otimes y^{\prime}\right\rangle\right| \leqslant \alpha(u)\left\|x^{\prime}\right\|\left\|y^{\prime}\right\|
$$

for all $x^{\prime} \in E^{\prime}, y^{\prime} \in F^{\prime}$ and $u \in E \otimes F$. If $\psi$ denotes the canonical embedding $E \otimes_{\varepsilon} F \rightarrow \mathcal{L}\left(E^{\prime}, F\right)$, then (3.3) ensures that $\psi$ is continuous, with the norm $\leqslant 1$. Hence, it can be extended by continuity to the completion, $\hat{\psi}: E \hat{\otimes}_{\varepsilon} F \rightarrow \mathcal{L}\left(E^{\prime}, F\right)$. 
Let $\mu$ be a Radon probability on $E \hat{\otimes}_{\alpha} F$. It is natural to put $\mu_{N \otimes M}:=$ $\pi_{N \otimes M}(\mu)$ in order to obtain a cylindrical measure on $E \otimes F$, but first we must be sure that the operator $\pi_{N \otimes M}: E \otimes F \rightarrow(E / N) \otimes(F / M)$ has a continuous prolongation to the space $E \hat{\otimes}_{\alpha} F$.

We can identify an element $\pi_{N \otimes M}(u) \in(E / N) \otimes(F / M)$ with the operator

$$
\left(\pi_{N \otimes M}(u)\right)^{\wedge}=\pi_{M} \circ \hat{u}^{\circ} \pi_{N}:(E / N)^{\prime} \rightarrow F / M
$$

Therefore, we can write $\pi_{N \otimes M}=\rho_{N, M} \circ \psi$ where $\rho_{N, M}: \mathcal{L}\left(E^{\prime}, F\right) \rightarrow \mathcal{L}\left((E / N)^{\prime}\right.$, $(F / M))$ is defined by

$$
\rho_{N, M}(w):=\pi_{M} \circ{ }^{\circ}{ }^{t} \pi_{N}
$$

Thus, since $\alpha$ satisfy (3.3)

$$
\hat{\pi}_{N \otimes M}(u):=\left(\rho_{N, M} \circ \hat{\psi}\right)(u)
$$

defines a mapping from $E \hat{\otimes}_{\alpha} F$ into $(E / N) \otimes(F / M)$ which prolongues $\pi_{N \otimes M}$.

Hence, if $\alpha$ satisfies (3.3) and $\mu$ is a probability on $E \hat{\otimes}_{\alpha} F$, then $\mu$ defines a cylindrical measure on $E \otimes F$ by

$$
\mu_{N \otimes M}:=\hat{\pi}_{N \otimes M}(\mu)
$$

We denote this cylindrical measure by $\breve{\mu}=\left(\mu_{N \otimes M}\right)$.

The inverse connection is more interesting for us.

Definition. Cylindrical measure $\lambda$ on $E \otimes F$ is a Radon probability on $E \hat{\otimes}_{\alpha} F$ if there exists a unique Radon probability $\mu$ on $E \hat{\otimes}_{\alpha} F$ such that $\mu^{\prime}=\lambda$.

The condition on uniqueness is essential. Namely, if it exists a probability $\mu$ on $E \hat{\otimes}_{\alpha} F$ such that $\lambda=\check{\mu}$, it cannot be assumed a priori that $\mu$ is unique (as in the classical case). A sufficient condition for this is due by Prohorov (cf. [11], Theorem 22, p. 81): it is sufficient that the mappings $\left\{\hat{\pi}_{N \otimes M}, N \in\right.$ $F C(E), M \in F C(F)\}$ separate points of $E \hat{\otimes}_{\alpha} F$.

Proposition 3.3. The following conditions are equivalent:

(i) $\left\{\hat{\pi}_{N \otimes M}, N \in F C(E), M \in F C(F)\right\}$ separate points of $E \hat{\otimes}_{\sigma} F$.

(ii) $\left\{u \mapsto\left\langle u, x^{\prime} \otimes y^{\prime}\right\rangle, x^{\prime} \in E^{\prime}, y^{\prime} \in F^{\prime}\right\}$ separate points of $E \hat{\otimes}_{\alpha} F$.

(iii) $\left\{u \mapsto\left[u, x^{\prime}\right], x^{\prime} \in E^{\prime}\right\}$ separate points of $E \hat{\otimes}_{\infty} F$.

(iv) $\hat{\psi}: E \hat{\otimes}_{\alpha} F \rightarrow \mathcal{L}\left(E^{\prime}, F\right)$ is one-to-one

Proof. Let us prove only (i) $\Rightarrow$ (ii). Take $u \in E \hat{\bigotimes}_{\alpha} F, u \neq 0$, and $N \in F C(E)$, $M \in F C(F)$ for which holds $\hat{\pi}_{N \otimes M}(u) \neq 0$, i.e. $\pi_{M} \circ \hat{u}^{t} \pi_{N} \neq 0$. There exist $x_{N}^{\prime} \in$ $(E / N)^{\prime}, y_{M}^{\prime} \in(F / M)^{\prime}$ such that 


$$
\left\langle\left(\pi_{M} \circ \hat{u} \circ^{t} \pi_{N}\right)\left(x_{N}^{\prime}\right), y_{M}^{\prime}\right\rangle \neq 0
$$

Define $x^{\prime}:={ }^{t} \pi_{N} x_{N}^{\prime}, y^{\prime}:={ }^{t} \pi_{M} y_{M}^{\prime} . \quad$ Then it holds $\left\langle u, x^{\prime} \otimes y^{\prime}\right\rangle \neq 0$.

In the sequel we will assume that $\alpha$ satisfies (3.3) and

$$
\hat{\psi}: E \hat{\otimes}_{a} F \rightarrow \mathcal{L}\left(E^{\prime}, F\right) \text { is one-to-one }
$$

Thus, from the well known conditions for the fulfilment of (3.3) and (3.4) we obtain

Proposition 3.4. Let $\alpha$ be a reasonable norm and $\lambda$ a cylindrical measure on $E \otimes F$. If $E$ or $F$ have the metric approximation property, and if it exists a probability $\mu$ on $E \hat{\otimes}_{\alpha} F$ for which holds $\mu=\lambda$, then $\mu$ is unique.

We will denote such probability by $\hat{\lambda}$. Hence, in this case we have $(\hat{\lambda})^{2}=\lambda$, $(\mu)^{\wedge}=\mu$.

\section{§4. Fourier Transform and Tmage of Cylindrical Measure}

Let $\lambda$ be a cylindrical measure on $E \otimes F$, and $w_{1}: E \rightarrow G, w_{2}: F \rightarrow H$ continuous linear operators. Denote $W:=w_{1} \otimes w_{2}$. First we define the image $W(\lambda)$.

Take $X \in F C(G)$. Then $N:=w_{1}^{-1}(X) \in F C(E)$ and all the operators in the following commutative diagram are continuous:

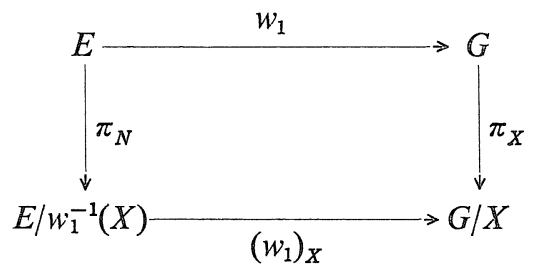

If we take now $Y \in F C(H)$, then $M:=w_{2}^{-1}(Y) \in F C(F)$ and the Radon probability $\lambda_{N \otimes M}$ on the space $\left(E / w_{1}^{-1}(X)\right) \otimes\left(F / w_{2}^{-1}(Y)\right)$ is well defined. Denote $W_{X \otimes Y}:=\left(w_{1}\right)_{X} \otimes\left(w_{2}\right)_{Y}$.

Definition. The image $\left(w_{1} \otimes w_{2}\right)(\lambda)$ of a cylindrical measure $\lambda$ is a cylindrical measure on $G \otimes H$ defined by

$$
\left(w_{1} \otimes w_{2}\right)(\lambda)_{X \otimes Y}:=W_{X \otimes Y}\left(\lambda_{N \otimes M}\right)
$$

The Fourier transform of a cylindrical measure is defined similarly as in the classical case: for $u^{\prime} \in E^{\prime} \otimes F^{\prime}$ and $s \in \mathbb{R}$ we define 


$$
\mathscr{F}(\lambda)\left(s u^{\prime}\right):=\mathscr{F}\left(u^{\prime}(\lambda)\right)(s)
$$

where $u^{\prime}(\lambda)$ is a probability on $\boldsymbol{R}$, defined in Proposition 3.2.

Proposition 4.1. For $w_{1} \in \mathcal{L}(E, G), w_{2} \in \mathcal{L}(F, H)$ it holds

$$
\mathscr{H}\left(\left(w_{1} \otimes w_{2}\right)(\lambda)\right)\left(u^{\prime}\right)=\mathscr{F}(\lambda)\left(\left({ }^{t} w_{1} \otimes{ }^{t} w_{2}\right)\left(u^{\prime}\right)\right)
$$

Proof. Let $u^{\prime}=\sum x_{j}^{\prime} \otimes y_{j}^{\prime}$ be a representation of $u^{\prime}$. We have

$$
\begin{aligned}
u^{\prime} \circ\left(w_{1} \otimes w_{2}^{\prime}\right) & =\left(\sum x_{j}^{\prime} \otimes y_{j}^{\prime}\right) \circ\left(w_{1} \otimes w_{2}\right) \\
& =\sum\left(x_{j}^{\prime} \circ w_{1}\right) \otimes\left(y_{j}^{\prime} \circ w_{2}\right) \\
& =\sum{ }^{t} w_{1} x_{j}^{\prime} \otimes{ }^{t} w_{2} y_{j}^{\prime}=\left({ }^{t} w_{1} \otimes{ }^{t} w_{2}\right)\left(u^{\prime}\right)
\end{aligned}
$$

and the Proposition follows.

The Fourier transformation establishes a one-to-one correspondence between cylindrical measures on Banach (more generally, locally convex) space $E$ and functions on $E^{\prime}$ of positive type, whose restrictions to the finite dimensional subspaces are continuous. This is an easy consequence of Bochner theorem (see [1], p. 19). The same proof gives:

Proposition 4.2. A function $\phi: E^{\prime} \otimes F^{\prime} \rightarrow C$ is the Fourier transform of a cylindrical measure $\lambda$ on $E \otimes F$ if and only if $\phi$ satisfies

(i) $\phi(0)=1$

(ii) $\phi$ is of positive type, i.e., for all $n \in \mathbb{N}, u_{1}^{\prime}, \cdots, u_{n}^{\prime} \in E^{\prime} \otimes F^{\prime}$ and $\zeta_{1}, \cdots$, $\zeta_{n} \in \mathbb{C}$ it holds

$$
\sum_{i, j=1}^{n} \zeta_{i} \bar{\zeta}_{j} \phi\left(u_{j}^{\prime}-u_{i}^{\prime}\right) \geqslant 0
$$

(iii) The restriction of $\phi$ to finite dimensional subspaces of $E^{\prime} \otimes F^{\prime}$ is continuous.

Lemma 4.3. For a cylindrical measure $\lambda$ on $E \otimes F$, the following is equivalent:

(i) $\left(x^{\prime}, y^{\prime}\right) \mapsto \mathscr{F}(\lambda)\left(x^{\prime} \otimes y^{\prime}\right)$ is continuous on $E^{\prime} \times F^{\prime}$.

(ii) If $\left(x_{j}^{\prime}\right)_{\gamma} \rightarrow x_{j}^{\prime}$ and $\left(y_{j}^{\prime}\right)_{\gamma} \rightarrow y_{j}^{\prime}(j=1, \cdots, n)$, then

$$
\mathscr{F}(\lambda)\left(\sum_{j=1}^{n}\left(x_{j}^{\prime}\right)_{\gamma} \otimes\left(y_{j}^{\prime}\right)_{\gamma}\right) \rightarrow \mathscr{F}(\lambda)\left(\sum_{j=1}^{n} x_{j}^{\prime} \otimes y_{j}^{\prime}\right)
$$

Proof. Denote $\phi:=\mathscr{F}(\lambda)$. From the classical inequality

$$
\left|\phi\left(u_{1}^{\prime}\right)-\phi\left(u_{2}^{\prime}\right)\right|^{2} \leqslant 2\left|1-\phi\left(u_{1}^{\prime}-u_{2}^{\prime}\right)\right|
$$

we obtain 


$$
\left|\phi\left(\sum_{j=1}^{n} u_{j}^{\prime}\right)-1\right| \leqslant \sum_{k=1}^{n}\left|\phi\left(\sum_{j=1}^{k} u_{j}^{\prime}\right)-\phi\left(\sum_{j=1}^{k-1} u_{j}^{\prime}\right)\right| \leqslant \sqrt{2} \sum_{k=1}^{n}\left|1-\phi\left(u_{k}^{\prime}\right)\right|^{1 / 2}
$$

Thus, it holds

$$
\begin{aligned}
& \left|\phi\left(\sum_{j=1}^{n}\left(x_{j}^{\prime}\right)_{\gamma} \otimes\left(y_{j}^{\prime}\right)_{\gamma}\right)-\phi\left(\sum_{j=1}^{n} x_{j}^{\prime} \otimes y_{j}^{\prime}\right)\right|^{2} \leqslant 2 \mid 1-\phi\left\{\sum_{j=1}^{n}\left(\left(x_{j}^{\prime}\right)_{\gamma}-x_{j}^{\prime}\right) \otimes\left(y_{j}^{\prime}\right)_{\gamma}\right. \\
& \left.\quad+\sum_{j=1}^{n} x_{j}^{\prime} \otimes\left(\left(y_{j}^{\prime}\right)_{\gamma}-y_{j}^{\prime}\right)\right\} \mid \leqslant 2 \sqrt{2} \sum_{k=1}^{n}\left\{\left|1-\phi\left[\left(\left(x_{k}^{\prime}\right)_{\gamma}-x_{k}^{\prime}\right) \otimes\left(y_{k}^{\prime}\right)_{\gamma}\right]\right|^{1 / 2}\right. \\
& \left.\quad+\left|1-\phi\left[x_{k}^{\prime} \otimes\left(\left(y_{k}^{\prime}\right)_{\gamma}-y_{k}^{\prime}\right)\right]\right|^{1 / 2}\right\}
\end{aligned}
$$

which converges to 0 if (i) is satisfied. The converse is obvious.

\section{§5. Type and Approximability}

A probability $\mu$ on a Banach space $G$ is of $\operatorname{order} p(0<p<\infty)$ if

$$
\|\mu\|_{p}:=\left\{\int_{G}\|z\|^{p} d \mu(z)\right\}^{1 / p}<\infty
$$

Let $\lambda$ be a cylindrical measure on $E \otimes F$. For $x^{\prime} \in E^{\prime}, y^{\prime} \in F^{\prime}$, the image $\left(x^{\prime}\right.$ $\left.\otimes y^{\prime}\right)(\lambda)$ is a probability on $\mathbb{R}$. We say that $\lambda$ is of type $p(0<p<\infty)$ if

$$
\|\lambda\|_{p}^{*}:=\sup \left\{\left\|\left(x^{\prime} \otimes y^{\prime}\right)(\lambda)\right\|_{p},\left\|x^{\prime}\right\| \leqslant 1,\left\|y^{\prime}\right\| \leqslant 1\right\}<\infty
$$

$\lambda$ is of type 0 if for every $\eta>0$ it exists $R>0$ such that

$$
\sup \left\{\left[\left(x^{\prime} \otimes y^{\prime}\right)(\lambda)\right](] R, \infty[),\left\|x^{\prime}\right\| \leqslant 1,\left\|y^{\prime}\right\| \leqslant 1\right\}<\eta
$$

The set of all cylindrical measures on $E \otimes F$ of type $p$ is denoted by $\mathscr{M}_{p}^{c}$ $(E \otimes F)$.

It is evident that a cylindrical measure of type $p_{1}$ is also of type $p_{2}$, for $p_{1}>p_{2}$.

The following proposition is obvious:

Proposition 5.1. Let $\lambda$ be a cylindrical measure of type $p>0$ on $E \otimes F$, $w_{1} \in \mathcal{L}(E, G), w_{2} \in \mathcal{L}(F, H)$ and $W=w_{1} \otimes w_{2}$. Then $W(\lambda)$ is a cylindrical measure of type $p$ on $G \otimes H$, and

$$
\|W(\lambda)\|_{p}^{*} \leqslant\left\|w_{1}\right\|\left\|w_{2}\right\|\|\|_{p}^{*}
$$

We say that a linear operator $W$ is $p$-Radonifying if it maps every cylindrical measure on $E \otimes F$ of type $p$ into a Radon probability of order $p$.

The following lemma establishes a connection between the type of a cylindrical measure and the continuity of its Fourier transform. The proof is identical to the classical one (cf. [1], p. 26) so we omit it:

Lemma 5.2. A cylindrical measure $\lambda$ on $E \otimes F$ is of type 0 if and only if 
the mapping $\left(x^{\prime}, y^{\prime}\right) \mapsto \mathscr{F}(\lambda)\left(x^{\prime} \otimes y^{\prime}\right)$ is continuous on $E^{\prime} \times F^{\prime}$.

Definition. A net $\left\{\lambda_{\gamma}, \gamma \in \Gamma\right\}$ of cylindrical measures converges $\otimes$-cylindrically to $\lambda \in \mathscr{M}^{c}(E \otimes F)$ if $\left(\lambda_{\gamma}\right)_{N \otimes M}$ converges to $\lambda_{N \otimes M}$ weakly, for every $N$ $\in F C(E), M \in F C(F)$.

The notion of $\otimes$-cylindrical convergence has also the sense for Radon probabilities on $E \hat{\otimes}_{\alpha} F$ :

Proposition 5.3. On $\mathscr{M}\left(E \hat{\otimes}_{\alpha} F\right)$ we have

(i) If $\mu_{\gamma} \rightarrow \mu$ weakly, then $\mu_{\gamma} \rightarrow \mu \otimes$-cylindrically

(ii) If $\mu_{\gamma} \rightarrow \mu \otimes$-cylindrically, then $\mu$ is unique.

Proof. (i) is immediate. If $\mu_{\gamma} \rightarrow \mu \otimes$-cylindrically, then $\breve{\mu}_{\gamma} \rightarrow \breve{\mu} \otimes$-cylindrically, and thus $\left(\mu_{\gamma}\right)_{N \otimes M} \rightarrow \mu_{N \otimes M}$ weakly. Let us suppose $\mu_{\gamma} \rightarrow \nu \otimes$-cylindrically. Then $\nu_{N \otimes M}=\mu_{N \otimes M}$ for all $N \in F C(E), M \in F C(F)$ so that $\nu=\check{\nu}$ and hence $\nu=\mu$, since $\alpha$ satisfies (3.4).

Proposition 5.4. If $w_{1} \in \mathcal{L}(E, G), w_{2} \in \mathcal{L}(F, H)$ and $\lambda_{\gamma} \rightarrow \lambda \otimes$-cylindrically, then $\left(w_{1} \otimes w_{2}\right)\left(\lambda_{\gamma}\right) \rightarrow\left(w_{1} \otimes w_{2}\right)(\lambda) \otimes$-cylindrically.

Proof. Denote $W=w_{1} \otimes w_{2}$. Let $W_{X \otimes Y}$ be the continuous linear operator defined in (4.1). By definition, $\left(\lambda_{\gamma}\right)_{N \otimes M} \rightarrow \lambda_{N \otimes M}$ weakly, where $N=w_{1}^{-1}(X)$, $M=w_{2}^{-1}(Y)$. Then,

$$
\left(W\left(\lambda_{\gamma}\right)\right)_{X \otimes Y}=W_{X \otimes Y}\left(\left(\lambda_{\gamma}\right)_{N \otimes M}\right) \rightarrow W_{X \otimes Y}\left(\lambda_{N \otimes M}\right)=(W(\lambda))_{X \otimes Y}
$$

The following lemma represents an essential step in the Radonification problem:

Lemma 5.5. Suppose $E^{\prime}$ and $F^{\prime}$ have the metric approximation property. If $\lambda$ is a cylindrical measure on $E \otimes F$ of type $p>0$, then there is a net $\left\{\lambda_{\gamma}\right\}$ of Radon probabilities on $E \otimes F$ (each of them is concentrated on some finite-dimensional subspace) such that $\left\{\lambda_{\gamma}\right\}$ converges $\otimes$-cylindrically to $\lambda$, and

$$
\left\|\lambda_{\gamma}\right\|_{p}^{*} \leqslant\|\lambda\|_{p}^{*}
$$

Proof. There exist finite dimensional operators $p_{\gamma}: E^{\prime} \rightarrow E^{\prime}$ and $q_{\gamma}: F^{\prime} \rightarrow F^{\prime}$ which converge pointwise to the identities, and it holds $\left\|p_{\gamma}\right\| \leqslant 1,\left\|q_{\gamma}\right\| \leqslant 1$. We can further suppose that $p_{\gamma}$ and $q_{\gamma}$ are weakly*-continuous [9]. Thus, there exist finite-dimensional operators ${ }^{t} p_{\gamma}: E \rightarrow E,{ }^{t} q_{\gamma}: F \rightarrow F$.

Define $\lambda_{\gamma}:=\left({ }^{t} p_{\gamma} \otimes{ }^{t} q_{\gamma}\right)(\lambda) . \quad \lambda_{\gamma}$ is a Radon probability, concentrated on some finite-dimensional space. Moreover, Proposition 5.1 gives 


$$
\left\|\lambda_{\gamma}\right\|_{p}^{*} \leqslant\left\|{ }^{t} p_{\gamma}\right\| \cdot\left\|^{t} q_{\gamma}\right\| \cdot\|\lambda\|_{p}^{*} \leqslant\|\lambda\|_{p}^{*}
$$

It remains to prove that $\lambda_{\gamma} \rightarrow \lambda \otimes$-cylindrically.

It is sufficient to obtain that $\mathscr{F}\left(\left(\lambda_{\gamma}\right)_{N \otimes M}\right)$ converges to $\mathscr{F}\left(\lambda_{N \otimes M}\right)$ uniformly on compact sets. Let $u^{\prime} \in((E / N) \otimes(F / M))^{\prime}$. Then

$$
\begin{aligned}
\mathscr{F}\left(\left(\lambda_{\gamma}\right)_{N \otimes_{M}}\right)\left(u^{\prime}\right) & =\mathscr{F}\left(\pi_{N \otimes M}\left({ }^{t} p_{\gamma} \otimes{ }^{t} q_{\gamma}\right)(\lambda)\right)\left(u^{\prime}\right) \\
& =\mathscr{F}(\lambda)\left(\left(p_{\gamma} \otimes q_{\gamma}\right)\left({ }^{t} \pi_{N \otimes M}\left(u^{\prime}\right)\right)\right.
\end{aligned}
$$

Take a representation ${ }^{t} \pi_{N \otimes M}\left(u^{\prime}\right)=\sum_{j=1}^{n} t_{j} x_{j}^{\prime} \otimes y_{j}^{\prime}$, where $\left\{x_{j}^{\prime} \otimes y_{j}^{\prime}\right\}$ is a basis of the space ${ }^{t} \pi_{N \otimes M}\left[((E / N) \otimes(F / M))^{\prime}\right] \subset E^{\prime} \otimes F^{\prime} . \quad x_{j}^{\prime}$ and $y_{j}^{\prime}$ can be taken such that $\left\|x_{j}^{\prime}\right\|=\left\|y_{j}^{\prime}\right\|=1$ holds.

Denote $\left(x_{j}^{\prime}\right)_{\gamma}:=p_{\gamma} x_{j}^{\prime},\left(y_{j}^{\prime}\right)_{\gamma}:=q_{\gamma} y_{j}^{\prime} . \quad$ Then $\left(x_{j}^{\prime}\right)_{\gamma} \rightarrow x_{j}^{\prime}$ and $\left(y_{j}^{\prime}\right)_{\gamma} \rightarrow y_{j}^{\prime}$.

By assumption, $\lambda$ is of type $p>0$, hence also of type 0. By Lemma 5.2, $\mathscr{F}(\lambda)$ is continuous on $E^{\prime} \times F^{\prime}$. Hence, by Lemma 4.2

$$
\mathscr{H}(\lambda)\left(\sum t_{j}\left(x_{j}^{\prime}\right)_{\gamma} \otimes\left(y_{j}^{\prime}\right)_{\gamma}\right) \rightarrow \mathscr{F}(\lambda)\left(\sum t_{j} x_{j}^{\prime} \otimes y_{j}^{\prime}\right)
$$

uniformly on bounded $\left\{t_{j}\right\}$. The lemma is proved.

\section{§6. Cylindrical Measures and $\tilde{p}$-summing Operators}

Let $(\Omega, \Sigma, P)$ be a probability space, and $f: \Omega \rightarrow E \hat{\otimes}_{\varepsilon} F$ such that $\omega \mapsto$ $\left\langle f(\omega), x^{\prime} \otimes y^{\prime}\right\rangle$ is measurable function, for all $x^{\prime} \in E^{\prime}, y^{\prime} \in F^{\prime}$. Define

$$
\|f\|_{p}^{*}:=\sup _{\substack{\left\|x^{\prime} \mid \leqslant 1\\\right\| y^{\prime} \| \leqslant 1}}\left\{\int_{\Omega}\left|\left\langle f(\omega), x^{\prime} \otimes y^{\prime}\right\rangle\right|^{p} d P(\omega)\right\}^{1 / p}
$$

and denote by $L_{p}^{*}\left(\Omega, \Sigma, P ; E \hat{\otimes}_{\varepsilon} F\right)$ the space of all such functions for which it holds $\|f\|_{p}^{*}<\infty$.

Proposition 6.1. Let $f \in L_{p}^{*}\left(\Omega, \Sigma, P ; E \hat{\otimes}_{\mathrm{\varepsilon}} F\right)$. If $W: E \otimes F \rightarrow G$ is $\tilde{p}$ summing, then

$$
\left\{\int_{\Omega}\|W(f(\omega))\|^{p} d P(\omega)\right\}^{1 / p} \leqslant \widetilde{\pi}_{p}(W)\|f\|_{p}^{*}
$$

Proof. Denote by $K_{1}$ the unit ball of the space $E^{\prime}$, with the weak topology $\sigma\left(E^{\prime}, E\right)$, similarly for $K_{2} \subset F^{\prime}$. By Pietsch Majorization theorem for $\tilde{p}$-summing operators (cf. [2], Theorem 1), there exists a Radon probability $\mu$ on the compact space $K:=K_{1} \times K_{2}$, such that for every $u \in E \otimes F$ it holds

$$
\|W(u)\| \leqslant \widetilde{\pi}_{p}(W)\left\{\int_{K}\left|\left\langle u, x^{\prime} \otimes y^{\prime}\right\rangle\right|^{p} d \mu\left(x^{\prime}, y^{\prime}\right)\right\}^{1 / p}
$$

Take $u \in E \hat{\otimes}_{\varepsilon} F$, and $\left\{u_{k}\right\} \subset E \otimes_{\varepsilon} F, u_{k} \rightarrow u$. Then $W(u)$ is well defined, since 
a $\tilde{p}$-summing operator can be extended by continuity on $E \hat{\otimes}_{\mathrm{g}} F$. Moreover, since the $\varepsilon$-norm topology on $E \otimes F$ is stronger than the weak topology $\sigma(E \otimes F$, $\left.E^{\prime} \otimes F^{\prime}\right)$, it holds $\left\langle u_{k}, x^{\prime} \otimes y^{\prime}\right\rangle \rightarrow\left\langle u, x^{\prime} \otimes y^{\prime}\right\rangle$ and, by Dominated Convergence theorem

$$
\int_{K}\left|\left\langle u_{k}, x^{\prime} \otimes y^{\prime}\right\rangle\right|^{p} d \mu\left(x^{\prime}, y^{\prime}\right) \rightarrow \int_{K}\left|\left\langle u, x^{\prime} \otimes y^{\prime}\right\rangle\right|^{p} d \mu\left(x^{\prime}, y^{\prime}\right)
$$

Thus, (6.2) holds for all $u \in E \hat{\otimes}_{\mathrm{e}} F$.

By Foubini's theorem, we have

$$
\begin{aligned}
& \left\{\int_{\Omega}\|W(f(\omega))\|^{p} d P(\omega)\right\}^{1 / p} \\
& \quad \leqslant \widetilde{\pi}_{p}(W)\left\{\int_{\Omega} \int_{K}\left|\left\langle f(\omega), x^{\prime} \otimes y^{\prime}\right\rangle\right|^{p} d \mu\left(x^{\prime}, y^{\prime}\right) d P(\omega)\right\}^{1 / p} \\
& \quad=\widetilde{\pi}_{p}(W)\left\{\int_{K} \int_{\Omega}\left|\left\langle f(\omega), x^{\prime} \otimes y^{\prime}\right\rangle\right|^{p} d P(\omega) d \mu\left(x^{\prime}, y^{\prime}\right)\right\}^{1 / p} \\
& \quad \leqslant \widetilde{\pi}_{p}(W) \sup _{\substack{\|\|^{\prime}\|\leq 1\\
\| y^{\prime}|| 1}}\left\{\int_{\Omega}\left|\left\langle f(\omega), x^{\prime} \otimes y^{\prime}\right\rangle\right|^{p} d P(\omega)\right\}^{1 / p} \\
& \quad=\widetilde{\pi}_{p}(W)\|f\|_{p}^{*}
\end{aligned}
$$

which proves (6.1).

Take now $\Omega=E \hat{\otimes}_{\mathrm{\varepsilon}} F, P=\mu, f: \Omega \rightarrow E \hat{\otimes}_{\mathrm{\varepsilon}} F$ identity. Then

$$
\|f\|_{p}^{*}=\sup _{\substack{\|\|^{\prime}\|\leq 1\\\| y^{\prime} \| \leqslant 1}}\left\{\int_{\Omega}\left|\left\langle u, x^{\prime} \otimes y^{\prime}\right\rangle\right|^{p} d \mu\left(x^{\prime}, y^{\prime}\right)\right\}^{1 / p}=\|\mu\|_{p}^{*}
$$

Thus, Proposition 6.1 gives:

Corollary 6.2. If $W: E \otimes F \rightarrow G$ is $\tilde{p}$-summing, $\mu$ a Radon probability on $E \hat{\otimes}_{\varepsilon}$ F of type $p$, then

$$
\|W(\mu)\|_{p} \leqslant \widetilde{\pi}_{p}(W)\|\mu\|_{p}^{*}
$$

Remark. The main difficulty in the proof above is crossing to the completion $E \hat{\otimes}_{\mathrm{g}} F$. This is necessary since the notion "Radon measure on $E \otimes F$ " has no sense ( $E \otimes F$ has no topology). But, if $\mu$ is concentrated on some finite dimensional space, and has the type $p$ (thus, $\mu \in \mathscr{M}_{p}^{c}(E \otimes F)$ ), then Corollary 6.2 remains true. We will apply this corollary only for such measures.

We are ready to prove the main result:

Theorem 6.3. Let $E, F, G, H$ be Banach spaces, $1<p<\infty$ and $\alpha$ a norm on $G \otimes H$ which satisfies (3.3) and (3.4). $W:=w_{1} \otimes w_{2}: E \otimes F \rightarrow G \hat{\otimes}_{\alpha} H$ is p-Radonifying if and only if it is $\tilde{p}$-summing, and for $\lambda \in \mathscr{M}_{p}^{c}(E \otimes F)$ it holds 


$$
\|W(\lambda)\|_{p} \leqslant \widetilde{\pi}_{p}(W)\|\lambda\|_{p}^{*}
$$

Remark. If $w_{1} \otimes w_{2}$ is $\tilde{p}$-summing, it is known that $w_{1}$ and $w_{2}$ must also be $p$-summing, and it holds $\tilde{\pi}_{p}(W)=\pi_{p}\left(w_{1}\right) \pi_{p}\left(w_{2}\right)$; moreover, if $w_{1}$ and $w_{2}$ are $p$-summing, then $w_{1} \otimes w_{2}$ is $\tilde{p}$-summing whenever the $\otimes$-norm $\alpha$ satisfies $\alpha \leqslant / d_{p}$ or $\alpha \leqslant g_{\dot{p}} \backslash$ (cf. [2], Theorem 3). See [7] for description of the norm $\mid d_{p}$ and $g_{p} \backslash$.

Another example of $\tilde{p}$-summing operators of the form $w_{1} \otimes w_{2}$ gives (cf. [2], Corollary 1):

Corollary 6.4. Let $w_{1}: E \rightarrow G$ be $p$-left-muclear, and $w_{2}: F \rightarrow H$-summing, $1<p<\infty$. Then $w_{1} \otimes w_{2}: E \otimes F \rightarrow G \hat{\otimes}_{d_{p}} H$ and $w_{2} \otimes w_{1}: F \otimes E \rightarrow H \hat{\otimes}_{g_{p}} G$ are p-Radonifying, and for $\lambda \in \mathscr{M}_{p}^{c}(E \otimes F)$ it holds

$$
\|W(\lambda)\|_{p} \leqslant g_{p}\left(w_{1}\right) \pi_{p}\left(w_{2}\right)\|\lambda\|_{p}^{*}
$$

Proof of Theorem 6.3: The only if part follows as in the classical case, cf. [12], Théorème 3.4, p. 196. It is sufficient to take a sequence $\left\{c_{n}\right\}$ of positive number of the sum 1 and $\left\{u_{n}\right\} \subset E \otimes F$ such that $Q_{p}\left(u_{n}\right)<\infty$. Denote by $\delta_{n}$ the Dirac measure in the point $c_{n}^{-1 / p} u_{n}$ and define $\lambda:=\sum c_{n} \delta_{n} . \lambda$ is obviously a cylindrical measure on $E \otimes F$ of type $p$ :

$$
\|\lambda\|_{p}^{*}=\sup _{\substack{\left\|x^{\prime}\right\| \leqslant 1 \\\left\|y^{\prime}\right\| \leqslant 1}}\left\{\Sigma c_{n}\left|\left\langle x^{\prime} \otimes y^{\prime}, c_{n}^{-1 / p} u_{n}\right\rangle\right|^{p}\right\}^{1 / p}=Q_{p}\left(u_{n}\right)
$$

Since $W$ is $p$-Radonifying, $W(\lambda)$ is a Radon probability on $G \hat{\otimes}_{a} H$ of order $p$.

$$
\begin{aligned}
\|W(\lambda)\|_{p} & =\left\{\int\|W(u)\|^{p} d \lambda(u)\right\}^{1 / p}=\left\{\sum c_{n}\left\|c_{n}^{-1 / p} W\left(u_{n}\right)\right\|^{p}\right\}^{1 / p} \\
& =N_{p}\left(W\left(u_{n}\right)\right)
\end{aligned}
$$

Thus, $Q_{p}\left(u_{n}\right)<\infty$ implies $N_{p}\left(W\left(u_{n}\right)\right)<\infty$ and $W$ is $\tilde{p}$-summing.

Let us prove the sufficiency. The operator $W$, being $\tilde{p}$-summing, has the factorization of the form (cf. [2], Theorem 2 and Theorem 3):

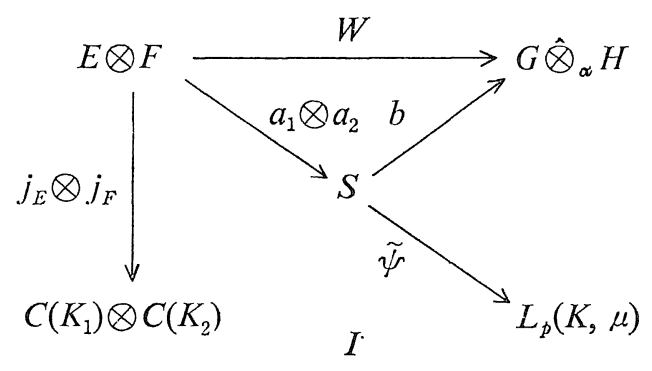


$K_{1}, K_{2}, K$ are defined in the proof of Proposition 6.1, $\mu_{1}$ and $\mu_{2}$ are the Pietsch measures, and $\mu:=\mu_{1} \otimes \mu_{2}$. The space $L_{p}(K, \mu)$ is obtained as the completion $L_{p}\left(K_{1}, \mu_{1}\right) \hat{\otimes}_{s_{p}} L_{p}\left(K_{2}, \mu_{2}\right)$, where $s_{p}$ is one of the norm $g_{p} \backslash$ or $/ d_{p}$ which coincides on the space $L_{p} \otimes L_{p}$ (cf. [8], Corollaire 4). $S$ is a closed subspace of $L_{p}(K, \mu)$, obtained as the closure of the space $S_{1} \otimes S_{2}$ in the norm $s_{p}$, where $S_{1}$ and $S_{2}$ are closed subspaces of $L_{p}\left(K_{1}, \mu_{1}\right)$ and $L_{p}\left(K_{2}, \mu_{2}\right)$, respectively. $\hat{\psi}=\psi_{1} \hat{\otimes} \psi_{2}$ is the canonical embedding, $b$ a continuous linear operator, with $\|b\| \leqslant \widetilde{\pi}_{p}(W)$ and $a_{1} \otimes a_{2}$ a $\tilde{p}$-summing operator. $j_{E}$ (and similarly $j_{F}$ ) is defined by $j_{E} x:=$ $\left(x^{\prime} \mapsto\left\langle x, x^{\prime}\right\rangle\right)$. $\quad I$ is defined by

$$
I\left(\sum f_{k} \otimes g_{k}\right):=\left(\left(x^{\prime}, y^{\prime}\right) \mapsto \sum f_{k}\left(x^{\prime}\right) g_{k}\left(y^{\prime}\right)\right)
$$

Let $\lambda$ be a cylindrical measure on $E \otimes F$ of type $p$. By Proposition 5.1 $\tilde{\lambda}:=\left(j_{E} \otimes j_{F}\right)(\lambda)$ is a cylindrical measure on $C\left(K_{1}\right) \otimes C\left(K_{2}\right)$ of type $p$. Since $C\left(K_{1}\right)^{\prime}$ and $C\left(K_{2}\right)^{\prime}$ have the metric approximation property, by Lemma 5.5 there exists a net $\left\{\lambda_{\gamma}\right\}$ of Radon probabilities on $C\left(K_{1}\right) \otimes C\left(K_{2}\right)$ (each of them is concentrated on some finite-dimensional space) which converges $\otimes$-cylindrically to $\lambda$ and

$$
\left\|\lambda_{\gamma}\right\|_{p}^{*} \leqslant\|\tilde{\lambda}\|_{p}^{*}=\left\|\left(j_{E} \otimes j_{F}\right)(\lambda)\right\|_{\dot{p}}^{*} \leqslant\left\|j_{E}\right\|\left\|j_{F}\right\|\|\lambda\|_{p}^{*}=\|\lambda\|_{p}^{*}
$$

The mapping $I$ is $\tilde{p}$-summing, with $\widetilde{\pi}_{p}(I) \leqslant 1$ (cf. [2], Lemma 1). Thus, Corollary 6.2 gives for the Radon probabilities $I\left(\lambda_{\gamma}\right)$ on $L_{p}(K, \mu)$ :

$$
\left\|I\left(\lambda_{\gamma}\right)\right\|_{p} \leqslant \widetilde{\pi}_{p}(I)\left\|\lambda_{\gamma}\right\|_{p}^{*} \leqslant\|\lambda\|_{p}^{*}
$$

Let $L_{p}(K, \mu)_{\sigma}$ be the space $L_{p}(K, \mu)$ with the weak topology. We can observe $I\left(\lambda_{\gamma}\right)$ as a Radon measure on $L_{p}(K, \mu)_{\sigma}$. By a version of Prohorov's theorem, see e.g. [4] Proposition 4, $\left\{I\left(\lambda_{\gamma}\right)\right\}$ is relatively compact in the topology of the weak convergence of probability measures. Hence, it exists a Radon probability $\nu$ on $L_{p}(K, \mu)_{\sigma}$ which lies in the closure of $\left\{I\left(\lambda_{\gamma}\right)\right\}$. We may suppose $I\left(\lambda_{\gamma}\right) \rightarrow \nu$ weakly, and hence also a $\otimes$-cylindrically. By Phillips theorem ([11], Theorem 3, p. 162) (weak and strong topology on a Banach space are Radonequivalent), $\nu$ is a Radon probability on $L_{p}(K, \mu)$.

On the other hand, $I(\tilde{\lambda})$ defines a cylindrical measure on $L_{p}\left(K_{1}, \mu_{1}\right) \otimes$ $L_{p}\left(K_{2}, \mu_{2}\right)$. Since $L_{p}$-spaces have the metric approximation property, by Proposition 3.4 there exists at most one Radon probability $I(\tilde{\lambda})^{\wedge}$ on $L_{p}(K, \mu) \simeq$ $L_{p}\left(K_{1}, \mu_{1}\right) \hat{\otimes}_{d_{p}} L_{p}\left(K_{2}, \mu_{2}\right)$. Since it holds $I(\tilde{\lambda})=\lim I\left(\lambda_{\gamma}\right)$, Proposition 5.3 ensures $\nu=I(\tilde{\lambda})^{\wedge}$, i.e. $I(\tilde{\lambda})=\check{\nu}$. Hence, $I(\tilde{\lambda})$ is a Radon probability on $L_{p}(K, \mu)$. We must show that $\nu$ is concentrated on $S$. 
Let $C)$ be a topology on $L_{p}(K, \mu)$ with the following basis of neighborhoods of zero:

$$
\begin{aligned}
& V=\left\{h \in L_{p}(K, \mu):\left|\left\langle h, f_{i}^{\prime} \cdot g_{j}^{\prime}\right\rangle\right|<\varepsilon, \quad \varepsilon>0,\right. \\
& \left.f_{1}^{\prime}, \cdots, f_{n}^{\prime} \in L_{p^{\prime}}\left(K_{1}, \mu_{1}\right), g_{1}^{\prime}, \cdots, g_{m}^{\prime} \in L_{p^{\prime}}\left(K_{2}, \mu_{2}\right)\right\}
\end{aligned}
$$

Since $f_{i}^{\prime} \cdot g_{j}^{\prime} \in L_{p^{\prime}}\left(K_{1} \times K_{2}, \mu_{1} \otimes \mu_{2}\right)$, the topology $C$ is weaker than the weak topology on $L_{p}(K, \mu)$. But, the converse is also true: take $h^{\prime} \in L_{p^{\prime}}(K, \mu)$. Since $L_{p^{\prime}}(K, \mu)$ is isometr ically isomorphic to the space $L_{p^{\prime}}\left(K_{1}, \mu_{1}\right) \hat{\otimes}_{s_{p^{\prime}}} L_{p^{\prime}}\left(K_{2}, \mu_{2}\right)$, $h^{\prime}$ can be approximated in $L_{p^{\prime}}$-norm by functions of the form $\sum_{i=1}^{n} f_{i}^{\prime} \otimes g_{i}^{\prime}$, $f_{i}^{\prime} \in L_{p^{\prime}}\left(K_{1}, \mu_{1}\right), g_{i}^{\prime} \in L_{p^{\prime}}\left(K_{2}, \mu_{2}\right)$, i.e., by the functions $(x, y) \mapsto \sum_{i=1}^{n} f_{i}^{\prime}(x) g_{i}^{\prime}(y)$, and our statement follows easily.

Consider the space $S:=S_{1} \hat{\otimes}_{s_{p}} S_{2} . \quad$ Let $N \in F C\left(L_{p}\left(K_{1}, \mu_{1}\right)\right), M \in F C\left(L_{p}\left(K_{2}, \mu_{2}\right)\right)$ be arbitrary. The norm $s_{p}$ satisfy (3.3), thus $\pi_{N \otimes_{M}}$ can be extended to a continuous linear operator $\hat{\pi}_{N \otimes_{M}}: L_{p}(K, \mu) \rightarrow\left(L_{p}\left(K_{1}, \mu_{1}\right) / N\right) \otimes\left(L_{p}\left(K_{2}, \mu_{2}\right) / M\right)$. Also, the Radon probability $\nu$ on $L_{p}(K, \mu)$ defines a unique cylindrical measure $\nu$ on $L_{p}\left(K_{1}, \mu_{1}\right) \otimes L_{p}\left(K_{2}, \mu_{2}\right)$. It holds $\hat{\pi}_{N \otimes M}(\nu)=\check{\nu}_{N \otimes M}=\pi_{N \otimes M}(\nu)$, and commutative diagram (6.6) shows that $\hat{\pi}_{N \otimes M}(\nu)$ is concentrated on $\pi_{N \otimes M}\left(S_{1} \otimes S_{2}\right)$.

Suppose that $\nu$ is not concentrated on $S$. Then it exists $h^{0} \notin S$ which lies in the support of $\nu$. Since $S$ is closed in the weak topology of the space $L_{p}(K, \mu)$, we can choose $\eta>0, f_{1}^{\prime}, \cdots, f_{n}^{\prime} \in L_{p^{\prime}}\left(K_{1}, \mu_{1}\right), g_{1}^{\prime}, \cdots, g_{m}^{\prime} \in L_{p^{\prime}}\left(K_{2}, \mu_{2}\right)$ such that it holds

$$
\left|\left\langle h-h^{0}, f_{i}^{\prime} \otimes g_{j}^{\prime}\right\rangle\right| \leqslant \eta
$$

for all $i, j$ and $h \in S_{1} \otimes S_{2}$.

Define $N \in F C\left(L_{p}\left(K_{1}, \mu_{1}\right)\right)$ by

$$
N:=\left\{f:\left\langle f, f_{i}^{\prime}\right\rangle=0, i=1, \cdots, n\right\}
$$

and similarly $M \in F C\left(L_{p}\left(K_{2}, \mu_{2}\right)\right)$. Suppose $\hat{\pi}_{N \otimes_{M}}\left(h^{0}\right) \in \pi_{N \otimes M}\left(S_{1} \otimes S_{2}\right)$. Then $\hat{\pi}_{N \otimes M}\left(h-h^{0}\right)=0$ for some $h \in S_{1} \otimes S_{2}$, hence $h-h^{0} \in N \otimes L_{p}\left(K_{2}, \mu_{2}\right)+L_{p}\left(K_{1}, \mu_{1}\right)$ $\otimes M$ which contradicts (6.7). Since $\hat{\pi}_{N \otimes M}\left(h^{0}\right)$ belongs to the support of $\hat{\pi}_{N \otimes M}(\nu)$ $\subset \pi_{N \otimes M}\left(S_{1} \otimes S_{2}\right)$, we get a contradiction again. Thus, $\nu$ is a Radon probability on $S$.

Finally, $W(\lambda)=b(\nu)$ is a Radon probability on $G \hat{\otimes}_{\alpha} H$, for which it holds

$$
\|W(\lambda)\|_{p} \leqslant\|b\|\|\nu\|_{p} \leqslant \widetilde{\pi}_{p}(W)\|\lambda\|_{p}^{*}
$$

The proof is complete.

Corollary 6.5. Let $1<p<\infty, \lambda \in \mathscr{M}_{p}^{c}\left(l_{1} \otimes l_{1}\right)$ and $i: l_{1} \rightarrow l_{2}$ be the canon- 
ical injection. Then $(i \otimes i)(\lambda)$ is a Radon probability on the space $H S\left(l_{2}, l_{2}\right)$ of Hilbert-Schmidt operators.

Proof. By Grothendieck's result, $i: l_{1} \rightarrow l_{2}$ is $p$-summing, for all $p>1$. By Theorem 6.3, $i \otimes i: l_{1} \otimes l_{1} \rightarrow l_{2} \hat{\otimes}_{g_{p}} l_{2}$ is $p$-Radonifying. This space coincides with the space of all $p$-summing operators: $l_{2} \rightarrow l_{2}$ (cf. [7], p. 91). The corollary follows since $p$-summing operator between Hilbert spaces is Hilbert-Schmidt operator-this is a known result of Pietsch-Pelczyński.

\section{References}

[1] Badrikian, A., Séminaire sur les Fonctions Aléatoires Linéaires et les Mesures Cylindriques, Lecture Notes in Mathematics 139, Springer-Verlag, 1970.

[2] Elezović, N., $\tilde{p}$-summing operators defined on tensor products of Banach spaces, to appear.

[3] — On some classes of nuciear operators defined on tensor products of Banach spaces, to appear.

[4] Maurey, B., Probabilités cylindriques, type et ordre. Applications radonifiantes, Séminaire Maurey-Schwartz 1972/73, Exposé I, 12p.

[5] — Rappels sur les operateurs sommants et radonifiants, Séminaire MaureySchwartz 1973/74, Exposé I-II, 9+10p.

[6] Pietsch, A., Operator ideals (in Russian), Mir, Moscow, 1982.

[7] Saphar, P., Produits tensoriels d'espaces de Banach et classes d'applications lineaires, Studia Math., 38 (1970), 71-100.

[8] — Applications $p$-decomposantes et $p$-absolument sommantes, Israel $J$. Math., 11 (1972), 164-179.

[9] Simons, S., If $E^{\prime}$ has the metric approximation property then so does $\left(E, E^{\prime}\right), M a t h$. Ann., 203 (1973), 9-10.

[10] Schatten, R., On direct product of Banach spaces, Trans. Amer. Math. Soc., 53 (1943), 195-217.

[11] Schwartz, L., Radon measures on arbitrary topological spaces and cylindrical measures Oxford University Press, Bombay, 1973.

[12] — Probabilités cylindriques et applications radonifiantes, J. Fac. Sci. Univ. Tokyo, Sec. I, A, 18 (1971), 139-286. 